\title{
ESCRITURA Y LECTURA: HECHO SOCIAL, NO NATURAL
}

WRITING AND READING: SOCIAL FACT, NOT NATURAL

\section{Volumen 13, Número 3 \\ Setiembre - Diciembre}

pp. $1-23$

Este número se publicó el 30 de setiembre de 2013

María de los Ángeles Carpio Brenes

Revista indizada en REDALYC, $\underline{\text { SCIELO }}$

Revista distribuida en las bases de datos:

CATÁLOGO DE LATINDEX, IRESIE, CLASE, DIALNET, DOAJ, E-REVIST@S, SHERPA/ROMEO, QUALIS, MIAR

Revista registrada en los directorios:

ULRICH'S, REDIE, RINACE, OEI, MAESTROTECA, PREAL, $\underline{\text { CLASCO }}$ 


\title{
ESCRITURA Y LECTURA: HECHO SOCIAL, NO NATURAL WRITING AND READING: SOCIAL FACT, NOT NATURAL
}

\section{María de los Ángeles Carpio Brenes ${ }^{1}$}

\begin{abstract}
Resumen: Este artículo de revisión bibliográfica tiene por objetivo exponer el marco histórico de la escritura y la lectura, la evolución de su instrucción desde los diferentes enfoques metodológicos que han surgido y la explicación que le ha dado la psicología a cómo aprende estas destrezas el ser humano. Este documento presenta esos temas en tres grandes apartados: sus orígenes como sistema de registro y control de ventas e intercambios, hasta la fuente de conocimiento que implica hoy día; las dos grandes tendencias metodológicas que han surgido en el proceso de instrucción a lo largo de historia; y los diferentes modelos cognitivos que han buscado explicar cómo se lleva a cabo el aprendizaje de la escritura y la lectura: se destaca el Modelo Dual Básico de Desarrollo Ortográfico como la teoría que mejor ilustra este proceso para aplicarlo en la práctica pedagógica actual.
\end{abstract}

Palabras clave: LECTOESCRITURA-APRENDIZAJE, LECTOESCRITURA-ENSEÑANZA Y MÉTODO, LECTOESCRITURA-HISTORIA

\begin{abstract}
This literature review paper aims to raise awareness of the historical context of writing and reading, the evolution of their instruction from the different methodological approaches that have emerged, and the explanation that psychologists have given to how human beings learn these skills. This paper presents these issues in three main sections: its origins as a system of registration and control of sales and exchanges, to the source of knowledge that entails today; the two major methodological trends that have emerged in the process of instruction along history; and the various cognitive models that have sought to explain how to carry out the learning of writing and reading: in which it is highlighted the Basic Dual Development Model Checker as the theory that best illustrates this process for application to current educational practice
\end{abstract}

Key words: READ-WRITE-LEARNING, READ-WRITE-TEACHING AND METHODS, READ-WRITESTORY

${ }^{1}$ Docente de la Escuela de Orientación y Educación Especial, Universidad de Costa Rica. Licenciada en Educación Especial y Máster en Psicopedagogía ambos títulos por la Universidad de Costa Rica. Doctora por la Universidad Autónoma de Madrid, España.

Dirección electrónica: maria.carpiobrenes@ucr.ac.cr

Artículo recibido: 8 de abril, 2013

Aprobado: 19 de agosto, 2013 


\section{Introducción}

El habla es la forma dominante de comunicación en el ser humano, sin embargo, conforme se fue organizando en sociedades, surgió la necesidad de comunicarse por otros medios que le permitieran recordar aquella información que podía quedar en el olvido con el pasar del tiempo. Fue así como surgió la escritura, con la intención de "comunicar a alguien por escrito algo" (Real Academia de la Lengua Española, 2001, s.p.), de transmitir a otros un mensaje por medio de un código diferente al oral. Paralelamente, nació la lectura, como el medio para decodificar esa información preservada en forma escrita.

Cada sociedad ha transmitido su sistema de escritura y de lectura como un "hecho social" porque ha sido traspasado a cada uno de sus ciudadanos de generación en generación. Como corresponde a una creación cultural y no a una habilidad natural, cada sociedad se preocupó por enseñar el código, es decir, por "el fomento de la capacidad de traducir o descifrar los símbolos escritos al lenguaje oral" (De Klerk y Simons, 1989, p. 20), así surgieron las primeras escuelas 4000 años atrás, con el objetivo principal de "enseñar la escritura cuneiforme a una clase social privilegiada, a unos 'especialitas': los escribas" (Bosco, 1995, p.31). Históricamente, se puede decir que hay escuela, antes que nada, para enseñar a leer, como lo declaró Charles Pinot Declos (1704-1772) "el que sabe leer sabe ya la más difícil de las artes" (p. 80).

Precisamente, por lo difícil que resulta para algunos el proceso de adquisición de la lectura y la escritura, es que la Psicología empezó a teorizar, a partir de la década de los años 60 , sobre su enseñanza, dada la centralidad de su aprendizaje en la escolarización, razón por la cual, en una reseña como ésta sobre la historia del código escrito, es necesario conocer también sobre los tipos de instrucción y las explicaciones que han surgido en torno a cómo aprende a leer y a escribir el ser humano.

\section{La escritura y la lectura como hecho histórico}

De acuerdo con Gaur (1990), los primeros pueblos que utilizaron la escritura fueron los egipcios, los sumerios, los chinos y los mesopotamios, quienes emplearon figuras y símbolos convencionales para representar sus ideas y pensamientos, sistema al que se le ha denominado ideográfico.

Los egipcios emplearon la escritura jeroglífica, compuesta de ideogramas, que son objetos que no tienen ningún sonido fonético; de fonogramas, signos que sirven para 
expresar la pronunciación; signos silábicos, que representan a varias consonantes; y determinativos, que sirven para mostrar su función semántica, como los verbos. El significado de este sistema sólo lo conocían los sacerdotes, pero cuando tenían que expresarse con mayor rapidez utilizaban signos más sencillos para representar las figuras y ser entendidos por un mayor número de personas. Esta especie de escritura taquigráfica tenía la ventaja de que no se necesitaba saber dibujar muy bien, por lo que fue evolucionando hasta sustituir los jeroglíficos.

La escritura de los chinos, destacable porque es el único sistema ideográfico antiguo que se mantiene actualmente, consta de cinco tipos de caracteres según el principio de su composición: El "pictográfico", basado en dibujos representativos; el "sencillo representativo", constituido por otros medios visuales lógicos, como los números, en los que una, dos o tres líneas horizontales, representan los números 1, 2 o 3; en el "compuesto representativo", los caracteres combinados expresan un concepto, como el símbolo del sol al lado del símbolo de la luna significa brillante; el grupo de los "homófonos" está formado por símbolos que se pronuncian de la misma manera pero tienen distinto significado, como el carácter de elefante que también se usa para dar el sentido de imagen porque ambas palabras se pronuncian xiàng. Finalmente, el grupo "semántico-fonético" está basado en la combinación de un carácter de significado junto con otro de pronunciación, por ejemplo, el carácter mujer combinado con el carácter pronunciado /mâ/, crea un carácter nuevo que significa madre. En el año 1958, el gobierno chino introdujo una escritura china romanizada conocida por el nombre de pinyin (ortografía fonética) como sistema oficial de escribir sonidos chinos y de transcribir los caracteres; con la política de "digrafía", se puede usar el pinyin o los caracteres, de acuerdo con las necesidades de quien escribe (Robinson, 1996).

Los sumerios inventaron la escritura cuneiforme, que consistía en escribir en tablillas de barro con un palo en forma de cuña (de ahí el nombre), combinaciones de números y dibujos de animales. Este tipo de escritura fue tomado por los babilonios, a quienes, en la región de Elam (sudeste de Irán) les sirvió para escribir el acadio y el elamita (lengua nosemítica ni Indoeuropea). Los persas llegaron posteriormente, se llevaron consigo la lengua y la escritura elamita y las convirtieron en instrumento de la administración. Al oeste, los urritas (lengua no-semítica ni indoeuropea) tomaron el sistema cuneiforme para escribir su lengua y a través de ellos llegaron a los hititas (indoeuropeos), quienes utilizaron el cuneiforme para su propia lengua y para la de los pueblos vecinos. Las tabletas de arcilla 
como soporte y el sistema cuneiforme se difundieron en toda el Asia menor, Siria y Anatolia, por lo comprensible que resultaba su sistema ideográfico (Ferreiro, 1996).

Sin embargo, su principal problema era que no todos sabían dibujar y tampoco era posible hacer representaciones gráficas de todo lo que se conoce. Los fenicios vieron esta dificultad 1200 años a.C. en estos pueblos del mediterráneo, por lo que se dieron a la tarea de perfeccionar ese sistema para su uso, creando la escritura fonética, en la cual cada signo no representa una idea, sino un sonido. Por sus actividades como navegantes y comerciantes, requerían de un sistema de escritura que les permitiera llevar un registro y un control de sus ventas e intercambios. Ellos se dieron cuenta de que con un solo símbolo podía representarse, por ejemplo, a un hombre o a un buey y que esta representación simbólica era comprensible para todos los pueblos con los que comerciaban. Esta misma representación podía tener otro significado, el de los sonidos; entonces, el símbolo con el que se representaba al buey, por ejemplo, pasó a ser el mismo para el sonido de la /a/, derivado de la palabra fenicia "aleph".

Al entrar en contacto los griegos con los fenicios en el siglo VIII a.C, estos ajustaron los sonidos de su idioma al alfabeto fenicio de 22 consonantes, agregándole dos signos más, debido a que la ausencia de las vocales en ese sistema les resultaba inadecuada para escribir poesía épica. Es por esto que, al ser griegas las primeras inscripciones de carácter alfabético descubiertas hasta ahora, muchos estudiosos consideran el griego como el primer alfabeto, término tomado del nombre de las dos primeras letras alpha y beta, documentado a partir del siglo III a.C. (Robinson, 1996).

El alfabeto griego se difundió por la costa oriental del mediterráneo, por todo occidente, hasta la Europa moderna, también por Oriente: a partir del arameo, hasta la India moderna. Por medio de las comunidades de la Iglesia Católica Romana y, más tarde, con la expansión colonial de Occidente. El alfabeto romano, que se diferencia del griego principalmente por las líneas curvas en sustitución de los trazos angulosos, se extendió hasta las tierras de América, Asia y África. Hoy en día, la mayor parte de los pueblos del mundo usan este sistema de escritura para representar los sonidos de sus lenguas, de manera que, con un número determinado de símbolos (como el llamado rotokas usado en las islas Salomón, el cual tiene 11 letras y el jmer, utilizado en Camboya, que se compone de 74 letras) y conociendo sus correspondencias sonoras, se pueden generar infinidad de palabras, dependiendo del grado de transparencia del sistema (Defior, 2006). 
Con el pasar del tiempo, la escritura dejó de ser un simple registro y control de ventas e intercambios y pasó a ser, según Cavallo y Chartier (1998), el medio ideal para transmitir el pensamiento del autor en un texto; la lectura se convirtió en el mecanismo por el cual el lector se apropia de la idea escrita, como se podrá ver en el recorrido histórico que hacen estos autores y que se sintetiza a continuación.

En la Época Antigua (siglo V a.C. al II d.C.), la escritura tenía como objetivo guardar la información, por lo que los textos se escribían en rollos de pieles de animales o en papiro, para poderlos conservar. La lectura era exclusiva de los eruditos y la modalidad que se practicaba era la de alta voz, para hacer comprensible al lector el sentido de una scriptio continua (palabras escritas una tras otra, sin espacios en blanco ni puntuación) inapreciable sin el sonido de la voz.

En la época Helenística (322 a.C. hasta 31 a.C.), permanecieron ciertas formas de oralidad, pero el libro pasó a desempeñar un papel fundamental, pues la literatura se enfocó en la composición, circulación y conservación de las obras. Se definió una estructuración más precisa, se estableció una medida estándar del volumen dentro de determinados extremos de altura y longitud; se establecieron sistemas de titulación y una serie de dispositivos para dividir los textos en partes y secciones. Aunque los libros estaban técnicamente predispuestos para la lectura, se conservaban en las bibliotecas como manifestaciones de grandeza de las dinastías en el poder.

En el año 31 a.C. Roma se apoderó de Grecia y el oriente griego. Sus libros se convirtieron en botines para las bibliotecas privadas romanas que se limitaban a los anales compilados por la casta sacerdotal y los testimonios documentales de archivo de la clase gentilicia. De su tradición literaria tomaron los modos de estructuración física del volumen literario y determinadas prácticas de lectura. En el siglo II a.C. surgió una creciente demanda de libros y de lectura, lo que provocó tres situaciones: la creación de bibliotecas públicas y el incremento de las privadas; el surgimiento de nuevos textos para franjas de lectores también nuevos, entre ellos los cristianos; y la producción y distribución de un tipo diferente de libro llamado el códice (libro con páginas), que logró su éxito por su menor coste, facilidad de distribución y lectura más libre de movimientos.

La manera como enseñaban a leer los romanos fue heredada de los griegos, quienes recurrían al alquiler de 24 esclavos que representaban los nombres de cada letra del alfabeto y se movían formando palabras, dirigidos por otro esclavo al que llamaban el pedagogo 
(Crowder, 1985). En el Espectáculo del alfabeto (en griego Grammatiké theoría), del poeta Atenience Callias, que data de la segunda mitad del siglo V a.C, se muestra un coro de 24 mujeres que personifican el alfabeto jónico, presentado en el prólogo de la manera siguiente: "Alfa, bêta, gamma, delta, ei, dsêta, êta, zêta, iota, kappa, lambda, mü, nü, xei, u, pei, rho, sigma, tau, ü, phei asiste, igual que chei, a psei- y hasta la". Luego el coro poniéndose de dos en dos, simula un ejercicio de escuela elemental "Beta alfa: ba; beta ei: bé; beta êta: bé; beta iota: bi; beta u: bu; beta ô: bô"; y luego en la antiestrofa: "Gamma alfa: ga; gamma ei: guê" y así sucesivamente lo cual supone en total 17 estrofas, cantadas en una sola y única melodía.

Los niños de la clase alta aprendían sobre todo las figuras y los nombres de las letras en riguroso orden alfabético, en ocasiones con ayuda de figuras de marfil u otros objetos similares. Escribían siguiendo el surco de las letras que el maestro había grabado en una tabla de madera, que después ellos mismos debían grabar con letras; las fases posteriores estaban constituidas por el trazado de sílabas, de palabras enteras y por último, frases. Los manuscritos se copiaban sin separación de palabras ni indicación de pausas dentro de los párrafos (scriptio continua).

El aprendizaje de la lectura, separado del de la escritura, se producía en un segundo momento. Los ejercicios iniciales de lectura tenían como base el conocimiento de las letras, después de sus asociaciones silábicas y de palabras completas; el adiestramiento continuaba con una lectura realizada lentamente durante largo tiempo, hasta que se llegaba poco a poco a una emendata velocitas, es decir, un considerable grado de rapidez sin incurrir en errores. El aprendizaje se hacía en voz alta, mientras la voz pronunciaba las palabras ya leídas, los ojos debían mirar las palabras siguientes, hecho que Quintiliano, que es la fuente de estos datos, consideró una operación dificilísima, pues requería "un desdoblamiento de la atención".

Durante la Edad Media (siglo V d.C. al XV d.C.), hubo grandes cambios en la cultura grecorromana. Surgió la lectura silenciosa o murmurada porque los libros se leían sobre todo para conocimiento de Dios y para la salvación del alma, por lo cual habían de ser entendidos, pensados, y hasta memorizados. Esta lectura requirió la separación de las palabras, el uso de convenciones gráficas, de signos distintivos que guiaban la vista entre las particiones del texto, como una práctica diversa de la puntuación que sirviera para facilitar el entendimiento de lo escrito. Se mantuvo la lectura en voz alta sólo en los espacios cerrados 
de las iglesias, los claustros, las escuelas religiosas y, algunas veces, en las cortes señoriales, donde se limitaba esta lectura solamente a las Sagradas Escrituras y a textos de edición espiritual.

En el Renacimiento (siglo XV a siglo XVI), la escritura se transformó en una suma de compendios con el fin de hacer más rápida la lectura: el espacio de la página se dividió en dos columnas estrechas, de modo que cada renglón entraba en un campo visual más fácil de captar. Nació el catálogo como instrumento para señalar la colocación de los libros en la biblioteca y el memoriale, una ficha en la que quedaban registrados los libros en préstamo, así que estos dejaron de ser acumulación patrimonial y pasaron a ser para lectura, pues se buscaba acumular los libros en el cerebro, por lo que revolucionó la lectura silenciosa, "intensiva”, de un grupo limitado y cerrado de libros transmitidos de generación en generación. Los maestros y escritores cristianos tomaron como estímulo para la lectura la salvación del alma, por lo que el libro de lectura elemental pasó a ser el salterio, cuyo conocimiento servía para comprobar si alguien sabía leer y escribir.

En la Edad Moderna (siglo XV d.C. a siglo XX), el libro no se vio transformado en sus estructuras esenciales: formato, género del texto, el momento y el modo de lectura; pero los modos de reproducción de los textos y de elaboración del libro cambiaron, con la invención de la prensa de imprimir, pues los costes de elaboración del libro bajaron considerablemente por la totalidad de ejemplares que se lograban en una tirada, asimismo, debido a que acortaba los tiempos de fabricación. Cada lector podía tener acceso a mayor número de libros, cada libro podía llegar a un número mayor de lectores. Esta transformación técnica favoreció la utilización diferenciada del mismo libro, pues podía ser leído en voz alta para los demás, o leído en silencio, para uno mismo. También propició la lectura "extensiva", permitiéndole al lector consumir numerosos, diversos y efímeros escritos que leía con rapidez y avidez; con una lectura libre, desenvuelta e irreverente, sometía a un examen crítico todo lo que leía (Cavallo y Chartier, 1998).

Esta transformación en las prácticas de la lectura provocó el interés y la necesidad, sobre todo a partir del siglo XVII, de una búsqueda de métodos que no sólo garantizaran el acceso a la lectura de un público lector que ahora incluía mujeres, niños y obreros (Viñao, 2009), sino que facilitaran el proceso de aprendizaje de la lectura, como los que se describen en el siguiente apartado (Lebrero, 1992). 


\section{Métodos de enseñanza de la lectura}

Desde el momento en que el aprendizaje de la lectura necesitó instrucción, aparecieron una serie de métodos didácticos empleados para su enseñanza. Sin embargo, todos se pueden clasificar en dos grandes categorías: los sintéticos o ascendentes, que comienzan por las unidades subléxicas -letras o sílabas- para terminar en la palabra; y los analíticos o descendentes, que comienzan por la frase o palabra y terminan en las sílabas y letras.

Los métodos sintéticos incluyen tres: alfabético, fonético y silábico; descritos por Bolaños, Cambronero y Venegas (2006) de la siguiente manera:

El método alfabético o método del deletreo fue el utilizado en los primeros días de Grecia y Roma hasta el fin de la Edad Media. Los y las estudiantes aprendían primero y por orden alfabético el nombre de las letras, mayúsculas y minúsculas; luego deletreaban y pronunciaban combinaciones de dos letras hasta que las aprendían bien y en seguida combinaciones de tres, cuatro y más letras, formando unidades silábicas o palabras; finalmente combinaban las sílabas y palabras para formar frases y oraciones breves. Solo después de numerosos ejercicios de repetición, el estudiante comenzaba verdaderamente a leer y, aun entonces, se daba mayor importancia al reconocimiento de palabras nuevas que a la comprensión del sentido del texto. La principal objeción al método es que el sonido del nombre de las letras no siempre indica la pronunciación de la palabra.

El método fónico fue propuesto en 1700 por la escuela francesa de lingüística de Port Royal, enmarcada en la corriente teológica del jansenismo francés, cuyo nombre proviene del teólogo y obispo católico Cornelio Jansen (1585-1638) -más tarde considerado herejepara quien la enseñanza de la lectura debía partir del conocimiento del sonido o estar asociado a cada letra o grafema individual, en vez de su nombre convencional. En la primera etapa del método, se enseñaban la forma y el sonido de las letras, empezando por las vocales, luego, el estudiante repetía el sonido varias veces a fin de establecer la asociación entre la letra y su forma. Después, se enseñaban las consonantes, según cierto orden establecido, y sus sonidos se combinaban con cada una de las vocales. Se practicaba el reconocimiento y pronunciación de series silábicas, enseguida, combinaciones de dos, tres, cuatro y más letras. Por último, las sílabas se combinaban en palabras y estas en frases y oraciones. Una de las objeciones al método es que el sonido de las consonantes puede darse con exactitud sólo en combinación con las vocales, ya que en forma aislada, puede provocar confusión. 
El método silábico es una derivación del método fonético, fue difundido en el siglo XVIII por Federico Gedike y Samiel Heinicke. Las unidades claves empleadas en la enseñanza de la lectura son las sílabas. Cuando se utiliza en relación con los idiomas alfabéticos, antes de presentar las sílabas, suelen enseñarse, con ayuda de palabras y cuadros, la forma y el sonido de algunas o de la totalidad de las vocales, las que se pronuncian primero como parte de una palabra o sílaba y, más tarde, en forma aislada. Si se utilizan cartillas preparadas conforme a métodos mecánicos, las sílabas se aprenden mediante repetidos ejercicios de reconocimiento y pronunciación. Otros tipos de cartillas de mejor calidad contienen cuadros que permiten establecer asociaciones significativas. Una vez pasada la primera lección, pueden hacerse ejercicios sobre la composición de frases, con palabras y sílabas ya enseñadas. Tales ejercicios ayudan a los y las estudiantes a descifrar las frases, o bien, la enseñanza de palabras puede continuar durante algún tiempo antes de enseñarles a leer frases. La principal crítica es que recarga demasiado la memoria del o la estudiante en las primeras etapas.

Los métodos analíticos son más recientes que los sintéticos, se remontan al año 1657 con la propuesta del método de palabra completa del reformista Jan Amós Comenius, padre de la pedagogía e introductor de las ilustraciones en los libros para aprendizaje gracias al uso de grabados en la imprenta. En esa fecha publicó Orbis Pictus, el primer libro para enseñar a leer, en el cual se mostraba, en un diseño arcaico, una estrategia moderna de enseñanza directa de palabras asociándolas con su significado, por medio de un dibujo que hacía referencia a los objetos nombrados, sin intervención de ningún principio alfabético, es decir, del recurso al nombre de las grafías (Viñao, 2009).

Sin embargo, fue Ovidio Decroly quien, antes de 1904, dio el impulso definitivo a los métodos analíticos con la propuesta del método global, el cual aplicaba en el Instituto de Enseñanza Especial de Bruselas y que se basaba en el sincretismo o percepción sincrética global que proponía la percepción de las cosas u objetos en su totalidad sin poder diferenciar las partes constitutivas de ese todo. Cada estudiante aprende a leer desde el principio las frases y palabras que surgen espontáneamente a través de las conversaciones escolares en torno a temas de interés, para posteriormente conocer y distinguir los elementos más simples que las componen, las sílabas y las letras. La metodología incluye sucesivas descomposiciones analíticas de dichas estructuras complejas: percepción global de la palabra y representación gráfica, lectura de la palabra, descomposición en sílabas; 
descomposición y recomposición de la palabra generadora, combinación de las sílabas ya conocidas para formar nuevas palabras y agrupación de las palabras en frases y oraciones. Sus detractores comentan que la lectura se hace muy lenta, que es causa de muchos problemas de aprendizaje y que es fácil no percatarse de los pequeños detalles de las letras.

En 1954, surge una propuesta de enseñanza analítica de relevancia en América Latina, el método natural integral, creado por la uruguaya Cledia de Mello, que establece como punto de partida el niño y la niña. Ella resalta que debe aprovecharse toda la experiencia vital y los aprendizajes que él y ella han ido acumulando desde su nacimiento. Por lo tanto, la enseñanza de la lectura y la escritura debe responder, desde sus comienzos, a la totalidad del vocabulario que la o el estudiante posee y a la capacidad que ya ha adquirido de expresar en forma oral sus pensamientos, sus sentimientos y sus percepciones en relación con los otros y su entorno. Sugiere tres pasos metodológicos: Primero, período de conversación, se trata de provocar la manifestación de las experiencias vitales de los niños y niñas, para que sirvan como el marco en el cual se desarrollará el aprendizaje sistemático de la lectoescritura. Segundo, el paso de la palabra hablada a la palabra escrita, en el cual la o el docente solicita al estudiantado que le digan alguna idea de las que expusieron y, entre todos, escogen el que desean que escriba en la pizarra (Molina y Chang, 1994). Finalmente, el período de producción mediante la palabra escrita y de ejercitación de la lectura, una vez dominado el abecedario completo en la etapa anterior, el grupo de estudiantes podrá expresar por escrito y leer todo aquello que ellos pueden pensar y expresar oralmente.

Otra exponente muy importante en nuestros días es la Dra. Emilia Ferreiro, psicóloga y pedagoga argentina que radica en México y quien se ha dedicado desde 1974 a investigar sobre los supuestos teóricos de la Psicogénesis del Sistema de Escritura de la niñez, antes y durante el proceso escolar. Según Martínez (2008), este no es un método de lectoescritura, sino un aporte teórico-pedagógico de las cinco fases del proceso de construcción de la lectoescritura: La fase simbólica, en la que el niño y la niña hacen una interpretación global de los nombres a partir del tamaño del objeto, estableciendo cierta relación a través de garabatos y dibujos. En la fase de escritura, buscan combinar las formas de las letras, es decir, intentan escribir. En la tercera fase o etapa silábica, están trabajando con sílabas, formulan hipótesis, escriben palabras de dos silabas y tres caracteres, relacionan lo oral con lo escrito y relacionan la escritura con el objeto (por ejemplo, escriben oio para representar ombligo). En el cuarto nivel de escritura, trabajan la hipótesis silábica para la alfabética, es 
un período de transición silábico-alfabética, establecen una relación entre sonidos y grafías, aunque todavía sus intentos de lectura son fallidos. En la última etapa, la alfabética, son capaces de comprender cada uno de los caracteres de la escritura y conocen letras de dos o más sílabas; además, construyen hipótesis de cantidad y variedad a partir de pautas sonoras y saben que las letras se representan por silabas y fonemas (por ejemplo, posa equivale a mariposa). De esta forma, a partir de los cinco años, el niño y la niña podrán iniciar su proceso formal de lectoescritura, si han tenido el estímulo adecuado.

Cuetos (2008) señala que entre los métodos sintéticos y los analíticos se encuentran los métodos mixtos, que se centran en que el reconocimiento de palabras, la comprensión y el descubrimiento de las correspondencias grafema-fonema se produzcan de forma combinada y se realimenten entre sí. Se destaca aquí el método ecléctico, que parte de la idea de que la enseñanza debe ir combinando los métodos de acuerdo con el desarrollo de los y las estudiantes, pues cada uno tiene sus propias características y necesidades muy particulares, por lo que es imprescindible utilizar diferentes procedimientos analíticos y sintéticos para enseñarles a leer y escribir. Bolaños, Cambronero y Venegas (2006), aclaran que en la actualidad sí existen numerosos procedimientos eclécticos; sin embargo, los que tienen especial significación combinan en un solo programa de enseñanza los métodos que estimulan una actitud reflexiva ante la lectura y una clara comprensión del significado de lo que se lee. Su principal crítica es que resulta complejo para aplicarlo en clases muy numerosas.

Con el fin, precisamente, de mejorar estas prácticas pedagógicas que surgieron en diferentes momentos y con enfoques diversos sobre cómo se enseña a leer, las investigaciones en psicología que se habían ocupado durante décadas del aprendizaje basado en la conducta observada, se enfocaron a partir de 1950, en el análisis de los procesos de la mente, especialmente los relacionados a cómo se aprende a leer.

Mayer (2002) comenta que, a pesar de que en los comienzos de la psicología educativa Huey (1908), en su influyente libro Psychology and Pedagogy of Reading, with a Review of the history of reading and writing and of methods, texts, and hygiene in Reading, resumió la importancia de los procesos de decodificación y comprensión lectora, no fue hasta los años 60 cuando nacieron los estudios sobre el aprendizaje de la lectura, que incluyen los procesos cognitivos básicos de almacenamiento de palabras, reconocimiento de fonemas, 
decodificación de palabras, acceso al significado de las palabras e integración de frases, cuyas explicaciones o modelos se han basado particularmente en el inglés.

\section{Modelos de aprendizaje de la lectura}

Como el código alfabético es una creación cultural, el cerebro humano no cuenta con la base biológica que le permita adquirir la lectura en forma natural, por lo que es indispensable su enseñanza. Los primeros modelos cognitivos que buscaban explicar el reconocimiento de palabras en la lectura inicial, plantearon que, ante el estímulo visual que es la palabra escrita, el niño y la niña deben realizar una categorización de los componentes de la palabra mediante dos rutas independientes, la ruta léxica o la ruta fonológica, términos utilizados inicialmente por Coltheart en 1978.

La lectura a través de la ruta léxica consiste en mirar la palabra, reconocer su configuración visual a partir de sus elementos gráficos o pictóricos más sobresalientes y acceder a su representación en el léxico mental, que contiene el conocimiento sobre el deletreo y la pronunciación del encadenamiento de los grafemas que forman las palabras. Leer en la ruta no léxica, involucra el uso de reglas de segmentación ortográfica hasta la segmentación fonológica, la recuperación o construcción del sonido y, después, el acceso al significado, cuando se trata de palabras conocidas.

Según Coltheart (2007), la vía léxica, directa o visual, implica una identificación inmediata de palabras conocidas, es decir, que ya han sido procesadas anteriormente y que están guardadas en el léxico mental, la vía fonológica, indirecta o subléxica, por su parte, es el mecanismo que convierte las palabras desconocidas o las pseudopalabras en sonidos y las secuencias de sonidos en palabras, mediante la aplicación de las reglas de correspondencia grafema-fonema. Pero, ¿cuál de las dos rutas se activa primero al acceder al significado de las palabras?

Como respuesta, surgen dos tendencias (Cuadrado, 2008). Por una parte, los que afirman que la ruta fonológica es predominante al inicio del aprendizaje de la lectura, pero una vez que se ha adquirido un dominio gradual del acceso indirecto, se reemplaza por una lectura más rápida gracias a la ruta visual (LaBerge and Samuels, 1974); por otra parte, quienes postulan que el acceso al significado de las palabras se realiza primero mediante la ruta visual, puesto que la representación semántica se obtiene a través de la representación 
ortográfica del léxico interno y la asociación grafema-fonema es posterior, es decir, que surge a partir de la enseñanza explícita de la lectura (Smith, 1971).

Sin embargo, con el tiempo, esta discusión sobre el funcionamiento independiente de las vías ha ido dando paso a una concepción del aprendizaje de la lectura que intenta explicar cómo ambas vías se usan y se desarrollan simultáneamente.

Recogiendo la amplia tradición de trabajos sobre aprendizaje de la lectura (Snowling y Frith, 1981; Liberman, 1973; Bryant y Bradley, 1983; Jiménez y Artiles, 1989; Maldonado, Sebastián y Soto, 1992; Sebastián y Maldonado, 1998; entre muchos otros), Alegría, Carrillo y Sánchez (2005) afirman que identificar una palabra consiste en establecer una conexión entre el léxico ortográfico, que es la identificación directa de esa palabra como parte del repertorio de palabras previamente memorizadas y el ensamblaje fonológico, que es un reconocimiento indirecto porque implica la traducción de los elementos ortográficos de la palabra a la forma fonológica correspondiente.

Otra defensora de esta idea es Sans (2008) quien sostiene que en el proceso lector, cada estudiante requiere tanto de la ruta fonológica para realizar la decodificación de las palabras mediante el desarrollo de la conciencia fonológica, como de la ruta léxica, para identificar la representación gráfica cuando se trata de una palabra conocida. Huerta y Matamala (1995) señalan que, mediante el sistema de correspondencia grafema-fonema, se analizan los grafemas asignándoles su fonema correspondiente y se llega, unas veces a la comprensión de la palabra y posteriormente a su pronunciación, otras veces, a una lectura sin comprensión de la palabra, como en el caso de la lectura de palabras desconocidas 0 pseudopalabra.

Otras teorías alternativas para explicar esta relación entre ambas rutas en el proceso de reconocimiento de palabras son la Hipótesis Visual-Fonológica (Ehri, 2006) y el modelo de lectura por Analogías (Goswami y Bryant, 2004).

Según Ehri (1995, 2006), las explicaciones basadas en el aprendizaje previo de las configuraciones visuales de las palabras para reconocerlas a través de la ruta léxica es insuficiente, porque recordar la forma ortográfica de cada palabra nueva que se adquiere de forma arbitraria plantea grandes exigencias en la memoria. En su opinión, es más plausible la hipótesis de que una vez que el niño y la niña han tenido conocimientos de las conexiones entre grafemas y fonemas, utilizarán estas claves para acceder a la representación ortográfica de la palabra almacenada en su memoria. Este modelo incluye un elemento 
fonológico a la ruta visual, que describe un recorrido Visual-Fonológico, que se da con base en el conocimiento de la correspondencia letra-sonido y la información ortográfica, los cuales, por medio de conexiones específicas inmediatas entre la forma visual de la palabra impresa (ortografía) y su pronunciación, almacenada en la memoria (representación fonológica), provocan, en esencia, que el lector "vea" la pronunciación de la palabra, lo cual crea vínculos directos entre la ortografía y su significado.

Por su parte, el modelo de Analogías de Goswami y Bryant (2004) plantea que el reconocimiento de la palabra se produce mediante un proceso basado en las analogías, al sugerir que la información fonológica que comparten dos o más palabras semejantes en su patrón ortográfico permite la deducción, a través de la rima, de reglas de correspondencia entre secuencias de letras y unidades fonológicas intrasilábicas. Así por ejemplo, al poder identificar las palabras "sol", "col" y "res", se facilita la lectura de "rol", pues las dos primeras comparten el final común (o rima) "ol" y la tercera comparte el ataque silábico "r". Por ello, la analogía se basa en la rima de las palabras y la secuencia de letras que conforman esa rima.

Tanto el modelo de doble vía como el de analogías inspiraron la formulación de una propuesta más reciente llamada Modelo de Procesamiento Dual Conexionista (Cuetos 2008), que sugiere la implementación de una vía léxica localista similar a la del modelo dual y una vía subléxica en la que se incluyen representaciones de los grafemas, cuyo punto de interacción entre las dos vías es el almacén de salida fonológica, una red competitiva en la que los códigos léxico y subléxico se unen para producir la pronunciación final de la palabra, permitiendo mejores resultados en la lectura de pseudopalabras.

Calvo y Carrillo (2011) refuerzan esta idea al afirmar que el lector experto es aquel que utiliza los dos procedimientos diferenciados en la decodificación, el reconocimiento directo de aquellas palabras que resultan familiares al lector por su frecuencia de uso en los textos escritos y el reconocimiento indirecto o de recodificación fonológica, que se aplica a palabras nuevas o poco familiares, o a palabras sin sentido o pseudopalabras, en las que no es posible un reconocimiento directo, porque depende del código alfabético y, por tanto, del conocimiento de la correspondencia fonema-grafema para la comprensión de la palabra.

Hasta aquí se han descrito, en forma muy general, los modelos de reconocimiento de palabras, sin embargo, es importante conocer también la forma en que se desarrolla esa lectura inicial en el niño y la niña, pues existen dos explicaciones: como proceso continuo y como desarrollo evolutivo. 
Las teorías de desarrollo continuo plantean que el aprendizaje de la lectura es tan complejo que sigue un proceso continuo y está sujeto a diversos factores. Un exponente a destacar es Perfetti (2001), quien explica que al principio del desarrollo las representaciones fonológicas y ortográficas están escasamente interconectadas. Con el tiempo, las conexiones entre ellas se refuerzan, de forma que llegan a estar redundantemente conectadas, gracias al aumento de conciencia fonémica y del creciente conocimiento de las reglas más complejas de decodificación. Para que se produzca un reconocimiento de palabras automático y eficiente es esencial que se produzca un aumento de la precisión en las correspondencias ortográficas y fonéticas y una redundancia en las conexiones fonológicas y ortográficas, por lo que la adquisición de representaciones altamente específicas y redundantes permitirá tanto la lectura léxica como la fonológica.

Por su parte, las teorías por etapas, es decir, los modelos discretos, también llamados evolutivos, proponen una serie de fases que se suceden, más o menos secuencialmente, en la adquisición de la lectura y cuya característica principal es la identificación de estrategias de lectura cualitativamente diferentes que definen cada uno de los estadios propuestos y que son adquiridos de forma ordenada y progresiva en función de las capacidades cognitivas y las influencias experienciales de los lectores (Carrillo y Marín, 1996).

Uno de los más conocidos es el Modelo Evolutivo de Uta Frith (1985), quien propone tres etapas en el desarrollo lector:

1. Etapa logográfica. El niño y la niña son capaces de identificar palabras escritas sólo por su apariencia visual, valiéndose del contexto donde aparecen y de la forma de su contorno (longitud, presencia de rasgos ascendentes o descendentes, la letra inicial) sin haber aprendido aún el código correspondiente para su descifrado. No es una lectura literal, pero accede a su significado reconociendo la palabra globalmente, como si se tratara de un dibujo; si cambiara la tipografía de esa palabra no podría reconocerla.

2. Etapa alfabética. En esta fase el niño y la niña aprenden a segmentar las palabras en las letras que las componen y a establecer la correspondencia entre las letras y los sonidos para formar palabras y frases. Es crucial en el aprendizaje de la lectura porque implica el uso del mecanismo de correspondencia grafema-fonema que es el que permite convertir el segmento ortográfico en fonológico para poder identificar palabras no familiares o incluso pseudopalabras. 
3. Etapa ortográfica. Supone la adquisición de un vocabulario más amplio a medida que el niño y la niña conocen nuevas palabras y las almacenan en la memoria que registra las palabras escritas llamada léxico ortográfico de entrada, que permite reconocer y reproducir las palabras de forma directa y rápida, sin tener que realizar la conversión letra-sonido.

De acuerdo con la autora, en un desarrollo normal, el niño y la niña deben pasar por las tres fases en orden secuencial, porque cada nuevo estadio es la combinación de las habilidades antiguas con las nuevas. Sin embargo, estudios posteriores señalan que las etapas evolutivas del desarrollo lector implican una serie de fases superpuestas que forman una estructura cada vez más compleja, no como estadios secuenciales, sino en términos de los componentes del modelo que están en proceso de formación y la naturaleza de la principal interacción con el desarrollo metalingüístico que tiene lugar simultáneamente a la adquisición del lenguaje escrito, tal y como lo propone el modelo de doble base de Seymour, Aro y Erskine (2003).

Este modelo sostiene que la adquisición de la alfabetización implica una interacción permanente entre el desarrollo del sistema ortográfico y las representaciones fonológicas, en las cuales las estructuras implícitas se hacen explícitas en respuesta a las demandas creadas por la estructura ortográfica. Los autores explican el desarrollo del lenguaje escrito mediante una representación esquemática con cajas y flechas (Figura 1), que simbolizan procesadores y conexiones unidireccionales y bidireccionales.

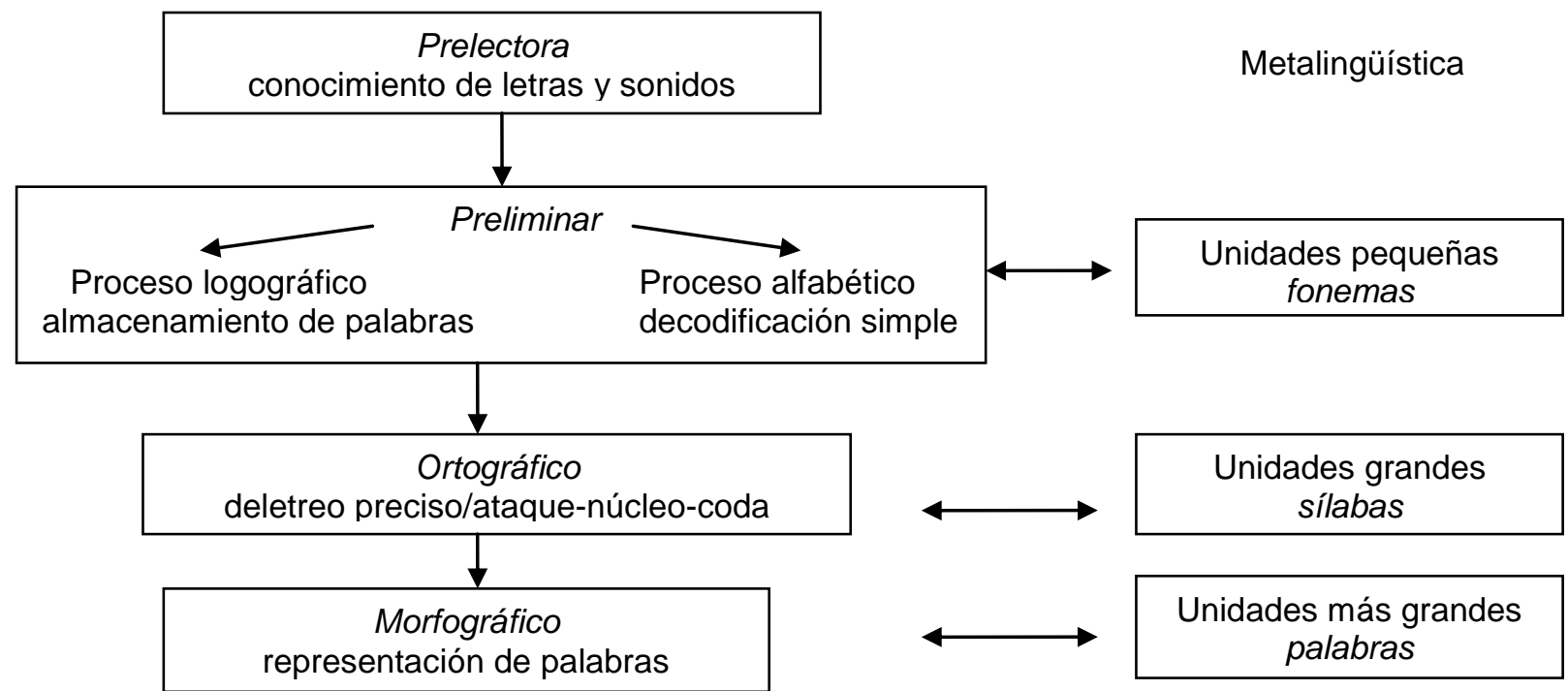

Figura 1. Representación esquemática del modelo dual básico del desarrollo ortográfico (Seymour, Aro y Erskine, 2003). 
Este esquema contempla cuatro fases para el desarrollo del sistema ortográfico: prelectora, preliminar, ortográfica y morfográfica (Seymour, 2007)

Fase 0, prelectora (pre-literacy). Corresponde al período anterior al aprendizaje de la lectura, en el que se ha desarrollado fundamentalmente el sistema lingüístico relacionado con el lenguaje oral. Se establece aquí el conocimiento básico de las letras del alfabeto y su correspondencia con los sonidos, sin un nivel metalingüístico porque, al no tener el niño suficientes demandas externas, la organización y control del lenguaje oral es aun inconsciente.

Fase 1, preliminar (foundation level). En esta etapa se establecen los elementos básicos del almacenamiento de palabras visualmente familiares (proceso logográfico) y la decodificación secuencial (proceso alfabético). El primero, según Rivas y Fernández (2007), es un sistema rudimentario de reconocimiento de palabras que se basa en las características visuales; mientras que el segundo facilita el reconocimiento de las formas visuales y motoras de los grafemas y su correspondencia con los fonemas.

Fase 2, ortográfica (orthographic level). Durante esta fase, se da una reorganización interna y se estructura el marco de las unidades lingüísticas para el deletreo preciso de las sílabas en torno a los elementos que forman las sílabas (ataque - núcleo - coda), o elementos de ataque y rima (núcleo - coda) que conforman las palabras almacenadas en la Fase 1. Se establecen en esta fase las reglas ortográficas, las relaciones entre unidades multigrafémicas y pronunciación, además de la relación entre deletreo y significado.

Fase 3, morfográfica (morphograpic level). En esta fase se da la formación de las representaciones de palabras complejas en las que se combinan las sílabas, además de que se añade la información sobre las formas multisilábicas y morfológicas complejas.

Esta secuencia de adquisición de la lectura es paralela a la evolución metalingüística, que representa la estructura segmental del lenguaje oral y sus funciones, con una influencia interactiva y causal sobre el desarrollo del lenguaje escrito. La dimensión temporal se representa mediante la forma en que se localizan espacialmente los sistemas en el esquema; así, los que están en la parte de arriba se desarrollan antes que los que están en la parte baja; las dobles flechas indican que los sistemas se influyen entre sí en su desarrollo y que todos van progresando de forma interrelacionada (Serrano, 2005).

Lo destacable del modelo de Seymour (2007) es la interacción que establece entre la adquisición de la lectura y el desarrollo metalingüístico como procesos simultáneos que 
deben ser sistemáticamente enseñados en el contexto de aprendizaje de la lectura. Defior y Serrano (2011) definen las habilidades metalingüísticas como la capacidad para tratar el lenguaje objetivamente, para reflexionar sobre él de forma explícita y manipular sus estructuras, fuera de su función comunicativa. Romero, Torrado y Mesa (2006) señalan que lo metalingüístico se convierte, según la lingüística, en un nuevo nivel de actividad del hablante, en el que no sólo el lenguaje se usa durante situaciones comunicativas, sino que, además, el individuo lo aísla del contexto inmediato para reflexionar, analizar y examinar sus diferentes dimensiones.

Las investigaciones actuales, principalmente en el campo de la psicolingüística, muestran que cuando el niño y la niña llegan al período de la lectura, aportan ya un gran número de las destrezas fonológicas, semánticas y ortográficas que proceden de su experiencia con el lenguaje oral, que se convierten, según Serrano (2005), en precursores del lenguaje escrito y predictores de la posterior adquisición lectora, lo que la convierte en una destreza indispensable en el aprendizaje de la lectura, pues

"si leer es enfrentarse a un sistema de escritura que refleja el lenguaje oral, todo profesional ha de tener en cuenta que el alumnado tiene que emparejar las unidades gráficas con sus correspondientes unidades sonoras. Para ello, se requiere, en primer lugar, tener habilidades para reflexionar conscientemente sobre los diferentes segmentos fonológicos del lenguaje oral, es decir, se ha de tener una adecuada conciencia fonológica. (Lozano y Lozano, 1999, p.137)

\section{Conclusiones}

A partir de lo expuesto en la historia de la lectura, de los métodos que han surgido para su enseñanza y de los modelos de aprendizaje que desde los años 60 se enfocaron en comprender cómo el cerebro aprende esta destreza, se explica la afirmación de que la lectoescritura es un hecho social y no una habilidad natural.

Como invento del ser humano, necesita de la instrucción para su adquisición. Durante muchos años, la tradición didáctica de la enseñanza de la lectura había puesto el énfasis en el método en sí, cuando en realidad la aplicación de un método no hace posible per se el aprendizaje de la lectura. El conjunto de estrategias que un método de enseñanza de la lectura incluye es lo que debe permitir la construcción de los procesos psicolingüísticos necesarios para que la y el estudiante analice el lenguaje y así adquiera la habilidad lectora. 
El consenso actual entre los investigadores del tema del aprendizaje de la lectura es que, para la enseñanza de esta destreza, es indispensable estimular dos procedimientos cognitivos diferenciados en el descifrado: la vía léxica o de reconocimiento visual y la vía subléxica o de recodificación fonológica. Mediante estas habilidades, la o el lector podrá adquirir la conciencia fonológica y las reglas de correspondencia grafema-fonema que son la base para el desarrollo del marco ortográfico y del morfográfico, indispensables para la lectura de sílabas y palabras.

En un idioma transparente como el español, se debe motivar a los y las docentes a utilizar el método que más se ajuste a las características de su lengua, como es el caso de métodos fonéticos en los hispanohablantes, porque a cada una de las 28 letras de su alfabeto le corresponde un sonido específico, con sólo tres excepciones: " $g$ " porque tiene el sonido /g/ y /x/; "c" con los sonidos /k/ y /s/; y "r" por sus sonidos /r/ y /r/. De esta forma, cuando el niño y la niña comprenden que a cada letra le corresponde un sonido y viceversa, habrá adquirido el código de su idioma, fundamental para el lector principiante, ya que le da la clave para realizar una lectura autónoma al permitirle identificar palabras nuevas usando como mecanismo para la decodificación las reglas de correspondencia grafema-fonema. Con el tiempo, la instrucción adecuada y el ejercicio constante, podrá adquirir la comprensión lectora y la composición escrita o redacción.

La decisión sobre qué método de lectura utilizar en la enseñanza de los niños y niñas va más allá de los intereses y gustos propios de cada docente, tiene que ver con el idioma, las características de aprendizaje de los y las estudiantes, el conocimiento teórico y práctico del método seleccionado, la necesidad de proponer actividades que desarrollen las habilidades psicolingüísticas en los y las estudiantes; pero, sobre todo, el compromiso docente por enseñar responsablemente, cumpliendo de manera sistemática los pasos del método escogido.

En el contexto actual, no es válido asumir una posición "ecléctica", entendida por algunos y algunas docentes como el tomar un poquito de este y aquel método y hacer el suyo propio, porque eso es en realidad, lo que en lenguaje coloquial se puede llamar un "arroz con mango", haciendo la analogía de lo insólito que resulta combinar estos dos elementos, igual sucede con algunos métodos de enseñanza de la lectura que no pueden mezclarse en la instrucción, por responder a tendencias cognitivas diferentes. 
Lo recomendable sería que los y las docentes conozcan profundamente los métodos de lectura existentes; investiguen la fundamentación teórica de cada uno a la luz de los modelos cognitivos actuales; analicen las estrategias que proponen para el desarrollo de las habilidades psicolingüísticas necesarias para el aprendizaje de la lectura y, una vez seleccionado el de su gusto, cumplan con la propuesta metodológica que se establece, sin dejar de lado la creatividad, al enriquecerlo con nuevas actividades, pero siguiendo siempre el principio teórico que lo sustenta.

\section{Referencias}

Alegría, Jesús, Carrillo, María, y Sánchez, Eloy. (2005). La Enseñanza de la lectura. Revista Investigación y Ciencia, 340, 6-14.

Bolaños, Bolivar, Cambronero, María y Venegas, Aracelly. (2006). Didáctica de la lectoescritura. Costa Rica: EUNED.

Bosco, Jim. (1995). Schooling and Learning in an Information Society. En U.S. Congress, Office of Technology Assessment, Education and Technology: Future Visions, OTA-BPEHR-169. Washington, DC: U.S. Government Printing Office.

Bryant, Peter y Bradley, Lynnette. (1983). Children’s reading problems. Oxford: Blackwell.

Calvo, Ángel y Carrillo, María. (2011). El acceso a la lectura desde la perspectiva cognitivapsicolingüística. Recuperado de http://www.educarm.es/lecto escritura/curso/03/t03.pdf

Carrillo, María y Marín, Javier. (1996). Desarrollo metafonológico y adquisición de la lectura: un programa de entrenamiento. Madrid: CIDE.

Cavallo, Guglielmo y Chartier, Róger. (1998). Historia de la lectura en el mundo occidental. Madrid: Santillana S.A. Taurus.

Coltheart, Max. (2007). Modeling Reading: The Dual-Route Approach. En Snowling, M y Hulme, Ch. The Science of reading a Handbook. (pp. 6-23). USA: Blackwell Publishing

Crowder, Robert. (1985). Psicología de la lectura. Madrid: Alianza Editorial.

Cuadrado, Isabel. (2008). Psicología de la Instrucción. Fundamentos para la reflexión y práctica docente. Francia: Editions Publibook

Cuetos, Fernando. (2008). Psicología de la lectura (7ª ed.). España: Wolters Kluwer.

De Klerk, Louis y Simons, Peter. (1989). Estudio de los procesos metacognitivos de la comprensión de lectura. En Fundación Germán Sánchez Ruiperez, Leer en la escuela. Nuevas tendencias en la enseñanza de la lectura (pp. 19-47). Madrid: Pirámide. 
Defior, Silvya, Fonseca, Liliana, Gottheil, Bárbara, Aldrey, Adriana, Jiménez, Grace, Pujals, María, Rosa, Graciela y Dolores, Francisca. (2006). LEE. Test de lectura y escritura en español. Buenos Aires: Paidós.

Defior, Silvya y Serrano, Francisca. (2011). Procesos fonológicos explícitos e implícitos, lectura y dislexia. Revista Neuropsicología, Neuropsiquiatría y Neurociencias, 11, 7994.

Ehri, Linnea. (1995). Phases of development in learning to read words by sight. Journal of Research in Reading, 18, 116-125.

Ehri, Linnea. (2006). The Roots of Learning to Read and Write: Acquisition o Letters Awareness. En David Dickinson y Susan Neuman, Handbook of Early Literacy Research (Volume 2, pp. 113-131). New York: Guilford Publications.

Ferreiro, Emilia (1996). Diversidad y proceso de alfabetización: de la celebración a la toma de conciencia. Recuperado de http://www.redescepalcala.org/inspector/DOCUMENTOS\%20Y\%20LIBROS/LECTURA/EMILIAFERREIRO-1.pdf

Frith, Uta. (1985). Beneath the Surface of Developmental Dyslexia. Recuperado de http://www.icn.ucl.ac.uk/dev group/ufrith/documents/Frith,\%20Beneath\%20the\%20surf ace\%20of\%20developmental\%20dyslexia\%20copy.pdf

Gaur, Albertine. (1990). Historia de la Escritura. Madrid: Pirámide.

Goswami, Usha y Bryant, Peter. (2004). Phonological skills and learning to read. New York: Phycgikigy Press Ltd.

Huerta, Elena y Matamala, Antonio. (1995). Tratamiento y prevención de las dificultades lectoras. Madrid: Visor Distribuciones S.A.

Huey, Edmund. (1908). Psychology and Pedagogy of Reading, with a Review of the history of reading and writing and of methods, texts, and hygiene in reading. Nueva York: McMillan.

Jiménez, Juan E. y Artiles, Ceferino. (1989). Cómo prevenir y corregir las dificultades en el aprendizaje de la lectura. Madrid: Síntesis.

LaBerge, David y Samuels, Jay. (1974). Toward a theory of automatic information processing in Reading. Cognitive Psychology, 6, 293-323.

Liberman, Isabelle. (1973). Segmentation of the spoken word and Reading acquisition. Bulletin of the Orton Society, 23, 65-77.

Lebrero, María Paz. (1992). Cómo formar buenos lectores. Madrid: Escuela Española. 
Lozano, Luis, y Lozano, Manuel. (1999). Evaluación y tratamiento de la dislexia fonológica. Aula Abierta, (74), 131-150. Recuperado de http://dialnet.unirioja.es/servlet/articulo?codigo $=45462$

Maldonado, Antonio, Sebastián, Eugenia y Soto, Pilar. (1992). Retraso en lectura: evaluación y tratamiento educativo. Madrid: Ediciones de la Universidad Autónoma de Madrid.

Martínez, José. (2008). Emilia Ferreiro y sus grandes aportes a la lectoescritura desmitificada de la escuela de hoy. Recuperado de http://www.elnuevodiario.com.do/app/article.aspx?id=107203

Mayer, Richard. (2002). Psicología de la educación. El aprendizaje de las áreas del conocimiento. Madrid: Pearson Educación.

Molina, Zaida y Chang, Sarita. (1994). El Método Natural Integral. Costa Rica: Editorial Fernández-Arce.

Perfetti, Charles. (2001). Reading Skill. En Neil Smelser and Paul Baltes (eds.), International encyclopedia of the social \& behabioral sciences (pp. 12800-12805). Oxford: Elsevier.

Real Academia Española. (2001). Escribir. En Diccionario de la lengua española (22 ${ }^{\circ}$ ed.). Recuperado de http://lema.rae.es/drae/srv/search?key=escribir

Rivas, Rosa y Fernández, Pilar. (2007). Dislexia, disortografía y disgrafía. Madrid: Ediciones Pirámide.

Robinson, Andrew. (1996). Historia de la escritura. Alfabetos, jeroglíficos y pictogramas. Barcelona: Ediciones Destino S.A.

Romero, Rita, Torrado, María, y Mesa, Carol. (2006). Emergencia de las capacidades metalingüísticas. Rev. Latinoam. Psicol., 38(3). Recuperado de http://pepsic.bvsalud.org/scielo.php?script=sci arttext\&pid=S0120$\underline{05342006000300002}$

Sans, Antonio. (2008). ¿Por qué me cuesta tanto aprender? Barcelona: Edebé.

Sebastián, Eugenia y Maldonado, Antonio. (1998). El desarrollo de las estrategias de segmentación de palabras en lectores jóvenes. En Jürgen M. Meisel (ed.), Adquisición del Lenguaje (pp. 82-96). Frankfurt: Klaus Dieter Verlag.

Serrano, Francisca. (2005). Disléxicos en español: papel de la fonología y la ortografía. (Tesis doctoral), Departamento de Psicologia Evolutiva y de la Educación, Universidad de Granada. Granada, España.

Seymour, Philip. (2007). Early Reading Development in European Orthographies. En Margaret Snowling y Charles Hulme (eds.), The Science of reading a Handbook (pp. 296-315). USA: Blackwell Publishing. 
Seymour, Philip, Aro, Mikko y Erskine, Jane. (2003). Foundation literacy acquisition in European orthographies. British Journal of Psychology, 94, 143-174.

Snowling, Maggie y Frith, Uta. (1981). The role of sound, shape and orthographic cues in early reading. British Journal of Psychology, 72, 83-87.

Smith, Frank. (1971). Understanding Reading. A Psycholinguistic Analysis of Reading and Learning to Read. Nueva York: Holt, Rinehart and Winston. [Edición española: Comprensión Lectora. México: Trillas, 1980.

Viñao, Antonio. (2009). La alfabetización en España: un proceso cambiante de un mundo multiforme. En Pedro Luis Moreno Martínez y Clotilde Navarro García (coords.), Perspectivas históricas de la educación de personas adultas (Vol. 3, N 1, pp. 5-19). Universidad de Salamanca. Recuperado de http://campus.usal.es/ efora/efora 03/articulos efora 03/n3 01 vinao.pdf 